\title{
Evaluating the role of strain ratio elastography in determining malignancy potential and calculating objective BIRADS US scores using ultrasonography and elastography features
}

\author{
Deniz Özel $\left.\right|^{1 A, B, C, D, E, F}$, Betül Duran Özel|2A,B,D,F \\ 'Sağlık bilimleri Unv Okmeydani Hospital, Interventional Radiology Clinic, İstanbul, Turkey \\ ${ }^{2}$ Sağlık bilimleri Unv Şişli Hamidiye Etfal Education and Research Hospital, Radiology Clinic, İstanbul, Turkey
}

\section{Abstract}

Purpose: The aims of this study were, firstly, to evaluate solid breast masses based on their malignancy potential and to determine whether the strain elastography ratio (SER) can contribute to classical grey-scale ultrasonography findings, and secondly, to define objective BIRADS US scores using ultrasound (US) and SER findings.

Material and methods: A total of 280 patients and 297 solid breast masses were evaluated using sonographic and elastographic data. The SER was measured for each lesion.

Results: The positive predictive values (PPV) for each criterion was calculated to be between $35 \%$ and $83.3 \%$. The lowest PPV was obtained from hypoechogenicity (35\%) and the highest PPV was obtained for anti-parallel features (83.3\%). The difference between the mean SER of benign and malignant lesions was statistically significant. After ROC analysis, the SER cut-off value was calculated to be 3.1 for determining if the mass was benign or malignant. Mass scores were calculated for each solid breast mass based on positive predictive values, and BIRADS US score was defined as the sum of mass scores.

Conclusions: SER findings can be used as malignancy criteria in evaluating solid breast masses. BIRADS US score can be objectively determined based on US and elastography features instead of doing subjective scoring. As an additional result, all solid breast masses have the possibility to be malignant, even though US and elastography findings indicate the opposite.

Key words: biopsy, large-core needle, breast neoplasms, elasticity imaging techniques, ultrasonography.

\section{Introduction}

Breast cancer is the most frequently diagnosed malignancy and the leading cause of cancer death among women worldwide [1]. The majority of breast cancers originate from solid masses. Although mammography (MG) and magnetic resonance (MR) findings are being used, when a solid breast mass is encountered, biopsy indications are predominantly determined from ultrasound (US) findings [2].
The reason for this is that US has an important potential in determining the malignancy likelihood for a lesion. A malignancy potential scoring system named BIRADS was described by the American College of Radiology. To use US findings only, a BIRADS US score was also defined. In this scoring method, the lesion's malignancy potential is subjectively determined $[3,4]$.

Newly introduced elastography methods may also contribute to this prediction by measuring the stiffness of the mass. There are two main methods of assessing stiff-

Correspondence address:

Deniz Özel, Sağlık bilimleri Unv Okmeydani Hospital, Interventional Radiology, Şişli İstanbul, 34384 Istanbul, Turkey, phone: 009053302749 08,

fax: 09021222178 00, e-mail: denizozel34@hotmail.com

Authors' contribution:

A Study design · B Data collection · C Statistical analysis · D Data interpretation · E Manuscript preparation · F Literature search · G Funds collection 
ness (elasticity) of a mass: strain elastography and shear wave elastography. In strain elastography, the stiffness of the mass can be viewed from the degree of strain (displacement) on manual compression. This technique is operator dependent and only gives a semi-quantitative estimation of tissue stiffness. With shear wave technology, the stiffness of a specific area is calculated by the device software without a comparison [5-7].

Currently, a significant proportion of breast biopsies are performed under US guidance in interventional radiology. This is because the method is real-time, easy to apply, and does not use ionising radiation [8-12].

Our aim in this study was to determine whether SER findings can contribute to US findings in evaluating solid breast masses and to define an objective US score using US-elastography findings.

\section{Material and methods}

A total of 280 patients and 297 solid breast masses were evaluated by performing USG-guided biopsy in our institute between October 2014 and September 2016.

Initially, all patients were evaluated with US. Transverse diameter, vertical diameter, border appearance (if there was microlobulation and spiculation), evidence of microcalcifications (MC), and acoustic transmission of solid breast masses were evaluated.

If the lesion's long axis parallel to skin/long axis perpendicular to skin ratio was greater than 1 , then this lesion was considered as lying parallel to the skin. Conversely, if the ratio was less than 1 , then this lesion was considered as lying anti-parallel to the skin. If the ratio was equal to 1 , it was excluded from evaluation. Anti-parallel placement was noted as a malignancy feature.

Acoustic transmission of the lesions was evaluated from their posterior acoustic property. If there was a posterior acoustic shadow, then it was noted as a malignancy feature.

Elastography strain ratio was measured for each lesion. The ratio was calculated automatically by the US device after sampling two regions of interest (ROI). The first ROI was obtained from the lesion and the other from the adjacent breast tissue. The ROI area of each lesion was as large as possible.

This study was approved by our Institute Research Ethics Committee.

\section{Core needle biopsy procedure}

Before the procedure, all patients were questioned for contraindications, such as anticoagulant medications or over-anxiety preventing core needle biopsy (CNB).

After necessary sterilisation was provided, topical anaesthetics were used to reduce pain at the procedure site.

The US devices were the Aplio 500 (Toshiba Medical Systems Europe) and the Hitachi Arietta V70 (Hitachi Med-
Table 1. Distribution of benign and malignant lesions

\begin{tabular}{|l|c|c|}
\hline Histopathology & Count & $\%$ \\
\hline Fibroadenoma & 109 & 51.9 \\
\hline Ductal epithelial hyperplasia & 17 & 8 \\
\hline Lipoma, lipogranuloma & 16 & 7.5 \\
\hline Mastitis & 13 & 6.1 \\
\hline Other: fibrocystic, benign adenomatoid changes & 56 & 26.4 \\
\hline Total & 211 & \\
\hline Invasive ductal carcinoma & 68 & 79 \\
\hline Invasive lobular carcinoma & 6 & 7 \\
\hline Carcinoma from distant sites & 2 & 2.3 \\
\hline Mucinous carcinoma & 7 & 8.1 \\
\hline Neuroendocrine carcinoma & 2 & 2.3 \\
\hline Sarcoma & 1 & 1.2 \\
\hline Total & 86 & \\
\hline
\end{tabular}

ical Systems Europa). The probe chosen was a 7.2-14 MHz linear probe. The probe was covered with a sterile instrument. All CNB procedures were performed with 14- or 16-gauge disposable and reusable needle systems. Range of fire was chosen as 15 and $22 \mathrm{~mm}$ according to the lesion and the patient conditions.

All procedures were performed by the same interventional radiologist with four years of experience.

After haemostasis was confirmed, evidence of bleeding under the skin was controlled with US. All patients were observed for a short period of time.

Histopathological results were obtained. Benign and malignant results were calculated as frequency in per cent (Table 1).

After all data was collected from all resources, then positive predictive values were calculated for the features considered as indicative for malignancy.

Mass scores were calculated based on positive predictive values for each lesion. Mass score was determined as an approximate value of PPV for each sonography - elastography feature (Table 2).

Finally, estimated BIRADS US scores were calculated according to the total mass score. The malignancy ratios were found to be completely concordant with the literature (Table 3).

\section{Statistical analysis}

Student's $t$-test was used to compare mean strain ratios for benign and malignant solid masses.

ROC analysis was used to determine the cut-off strain ratio point.

IBM SPSS Statistics program version 22 was used for statistical analysis. 
Table 2. Positive predictive values (PPV) for malignant, ultrasonography (USG), and strain wave elastography criteria

\begin{tabular}{|l|c|c|c|c|}
\hline USG - elastography criteria & True positive & Total positive & PPV \% & Mass score \\
\hline Anti-parallel placement to skin & 65 & 78 & 83.3 & 0.8 \\
\hline Spiculation-microlobulation & 97 & 122 & 79.5 & 0.8 \\
\hline Posterior acoustic shadow & 61 & 88 & 69.3 & 0.7 \\
\hline Hypo echo dominance & 91 & 256 & 35.5 & 0.35 \\
\hline Presence of microcalcifications & 34 & 46 & 73.4 & 0.7 \\
\hline Strain-ratio over cut-off value & 53 & 75 & 70.6 & 0.7 \\
\hline
\end{tabular}

Table 3. Malignancy ratios via BIRADS score and comparison with the literature

\begin{tabular}{|l|c|c|c|c|c|}
\hline Mass score total & EBS & Malignant & Benign & Malignity ratio (\%) & Literature \\
\hline $0-0.49$ & 3 & 2 & 111 & 1.8 & $0-2$ \\
\hline $0.5-1.49$ & $4 a$ & 4 & 38 & 9.5 & $2-10$ \\
\hline $1.5-2.49$ & $4 b$ & 41 & 58 & 41.4 & $10-49$ \\
\hline $2.5-3.49$ & $4 c$ & 31 & 4 & 88.6 & $50-95$ \\
\hline $3.5-4.2$ & 5 & 8 & 0 & 100 & $95-100$ \\
\hline Total & & 86 & 211 & & \\
\hline
\end{tabular}

EBS - Estimated BIRADS score

\section{Results}

This study included 280 patients and 297 solid breast masses. There were 278 females as compared to 2 males in the study. The average age was 45.6 years (range from 19 to 79 ).

Eighty-six of 297 solid breast masses were malignant (29\%) and the remaining 211 were benign. The most frequently seen benign tumour was fibroadenoma. The number of proven fibroadenomas was 109 with a percentage of 51.9. Other benign results could be determined as ductal epithelial hyperplasia, lipoma, granuloma, mastitis, etc. On the other hand, the most frequent malignant tumour was invasive ductal carcinoma, as expected. The total number was 68 , corresponding to $79 \%$. Subsequent malignant tumour types were invasive lobular carcinoma and mucinous carcinoma, with equal frequencies. Distribution of benign and malignant solid breast masses according to their frequencies is shown in Table 1.

Positive predictive values (PPV) were calculated based on US and elastography features after considering malignancy. The highest PPV was obtained from the anti-parallel placement feature at $83.3 \%$, whereas the lowest value was from hypo-echo dominance at $35 \%$. Table II shows the positive predictive values and mass scores of the sonographic features obtained in our study.

Figure 1 and Figure 2 show solid breast masses with their mass score as benign and malignant examples.

The mean strain ratio for benign lesions was calculated as $2.1 \pm 1.6$ (mean \pm standard deviation), whereas the mean strain ratio for malignant lesions was $4.6 \pm 2.6$.
The difference between the mean strain ratio of benign and malignant lesions was statistically significant $(p<0.01)$. In ROC analysis, the cut-off value was calculated to be 3.1, and the positive predictive value of strain ratio for malignant lesions was calculated to be 70.6 (Figure 3).

\section{Discussion}

The majority of breast core needle biopsies are performed under the guidance of US. Conventional radiologists tend to use MRI guidance when US findings are suspicious-negative. MRI is also an expensive modality and requires special equipment. On the other hand, MG is uncomfortable for many patients and uses ionising radiation. MG guidance can also be considered when microcalcifications accompany the suspicious mass or if they are the single positive sign. Finally, US is the single modality allowing real-time guidance, making it the preferred method.

The CNB procedure was used to obtain samples for histopathological diagnosis. CNB is the preferred procedure for solid breast masses, and the results are very convincing [13]. Brenner et al. [14] stated that there was a 99\% sensitivity ratio using 14-gauge needles and five samples for each lesion from their multicentre retrospective study.

When our histopathological findings were evaluated, as the first result, half of all benign lesions were fibroadenomas (52\%), corresponding to the literature. The most frequent malignant solid mass was invasive ductal carcinoma. Sixty-eight invasive ductal carcinomas were defined, which was $79 \%$ of all malignant diagnoses. This result was also 


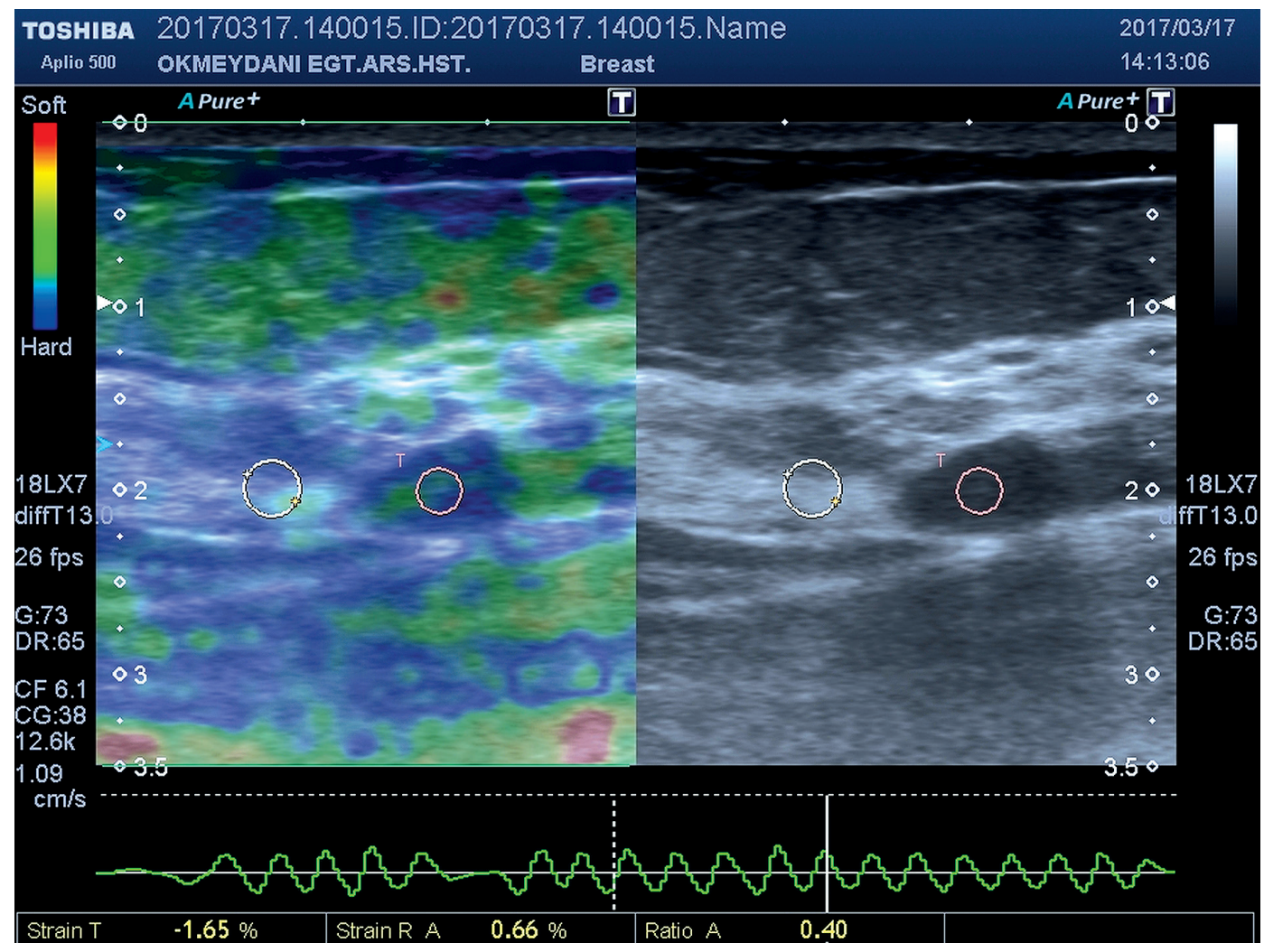

Figure 1. 35-year-old female. $7 \times 4 \mathrm{~mm}$ sharp borders, parallel placement. No posterior acoustic change. Strain ratio: 0.4. Mass score: 0.35. EBS: 3 . Diagnosis: Fibroadenoma

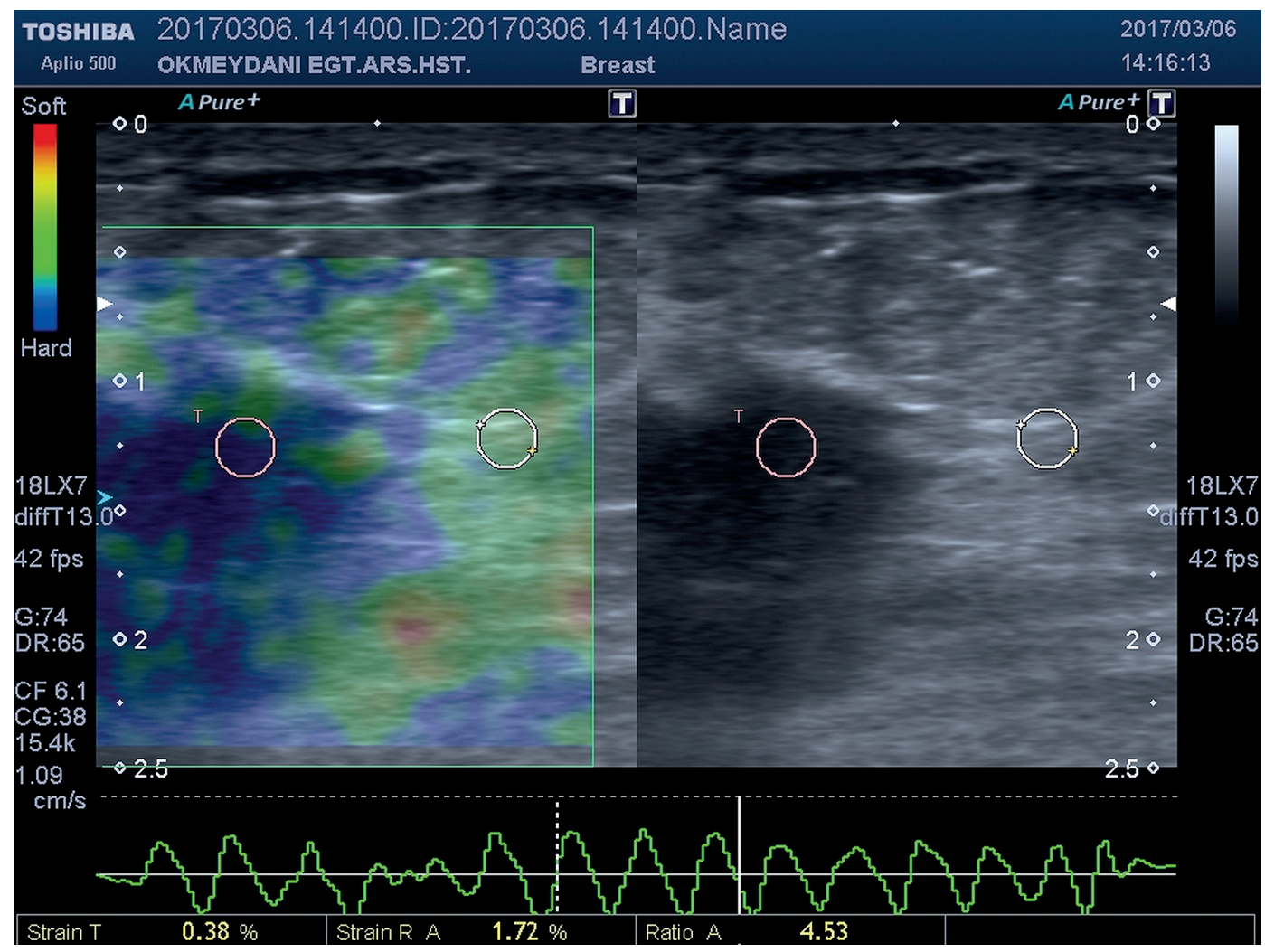

Figure 2.60-year-old female. $14 \times 18 \mathrm{~mm}$ irregular borders anti-parallel placement. No posterior acoustic change and microcalcification. Strain ratio: 4.53 . Mass score: $0.35+0.8+0.8+0.7=2.65$. EBS: 4 c. Diagnosis: Invasive ductal carcinoma 


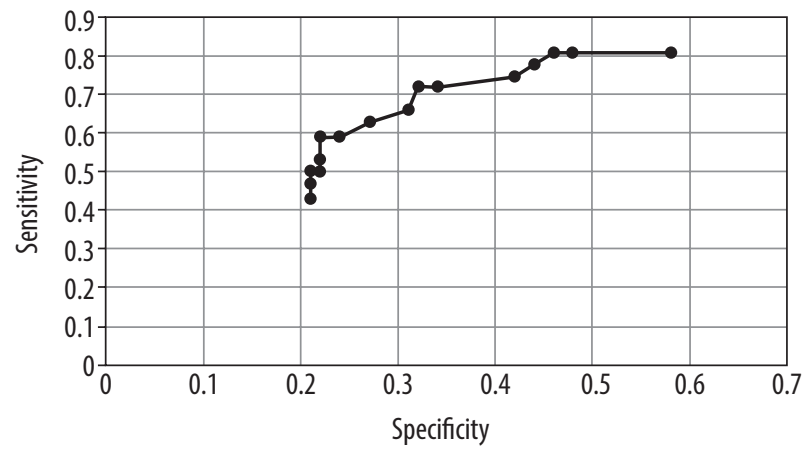

Figure 3. The ROC curve obtained from elastography strain ratio values

similar to the literature; around $80 \%$ of all breast cancers are invasive ductal carcinomas [15].

Our study included 278 females and two male patients. Breast carcinomas are found in women a hundred times more frequently than in men. Most of the time, male patients consulting an interventional radiology department complain about gynecomastia and solid breast masses are rarely seen. Only one male patient had invasive ductal carcinoma in our study. Invasive ductal carcinoma is the most frequently seen malignant tumour in males, as in females [16,17].

In order to define the BIRADS US score as a first step, the PPV was calculated for features indicative of malignancy. The PPV of anti-parallel placement to the skin was $83.3 \%$, and the number of lesions having this feature was 65. Parallel or anti-parallel placement to the skin surface by the long axis of the mass is an important feature in terms of benign-malignant differentiation. When the course of the mass is parallel to the skin, it favours benignity, while, conversely, anti-parallel placement indicates spreading of the lesion throughout the tissue planes. Studies on the PPV of the anti-parallel position for malignancy showed values of $69-81.2 \%$ [18-20].

The spiculated border feature showed a PPV of $79.5 \%$, and 97 of the lesions matched the criteria. Stavros et al. [18] and Constantini et al. [19] evaluated spiculation and microlobulation separately. Stavros et al. reported PPV values of $91.8 \%$ and $48.2 \%$ and Constantini et al. reported $87.5 \%$ and $100 \%$, respectively. Hong et al. [20] calculated the PPV for microlobulation and reported an $86 \%$ ratio. Radial protrusions characterised with spiculated margins extending from the mass have one of the highest PPV in terms of sonographic findings of malignancy. Border spiculation of the mass results from tumour cells infiltrating into the surrounding tissue. Because of the similar density of the hypoechoic spiculation and its extending fibrous tissue surrounding the malignant mass, hypoechoic spiculation may be indistinguishable on mammograms. For these cases US is very helpful.

Evidence of a posterior acoustic shadow indicates poor acoustic transmission and is considered as a malignancy criterion. The PPV was found to be $69.3 \%$ for this criterion in our study, and 61 of the lesions had this feature. Presence of posterior acoustic shadow due to less attenuation of the sound beam indicates that the mass could be malignant. The PPV values vary between $64.9 \%$ and $78.9 \%$ in terms of a malignancy indicator $[18,19]$.

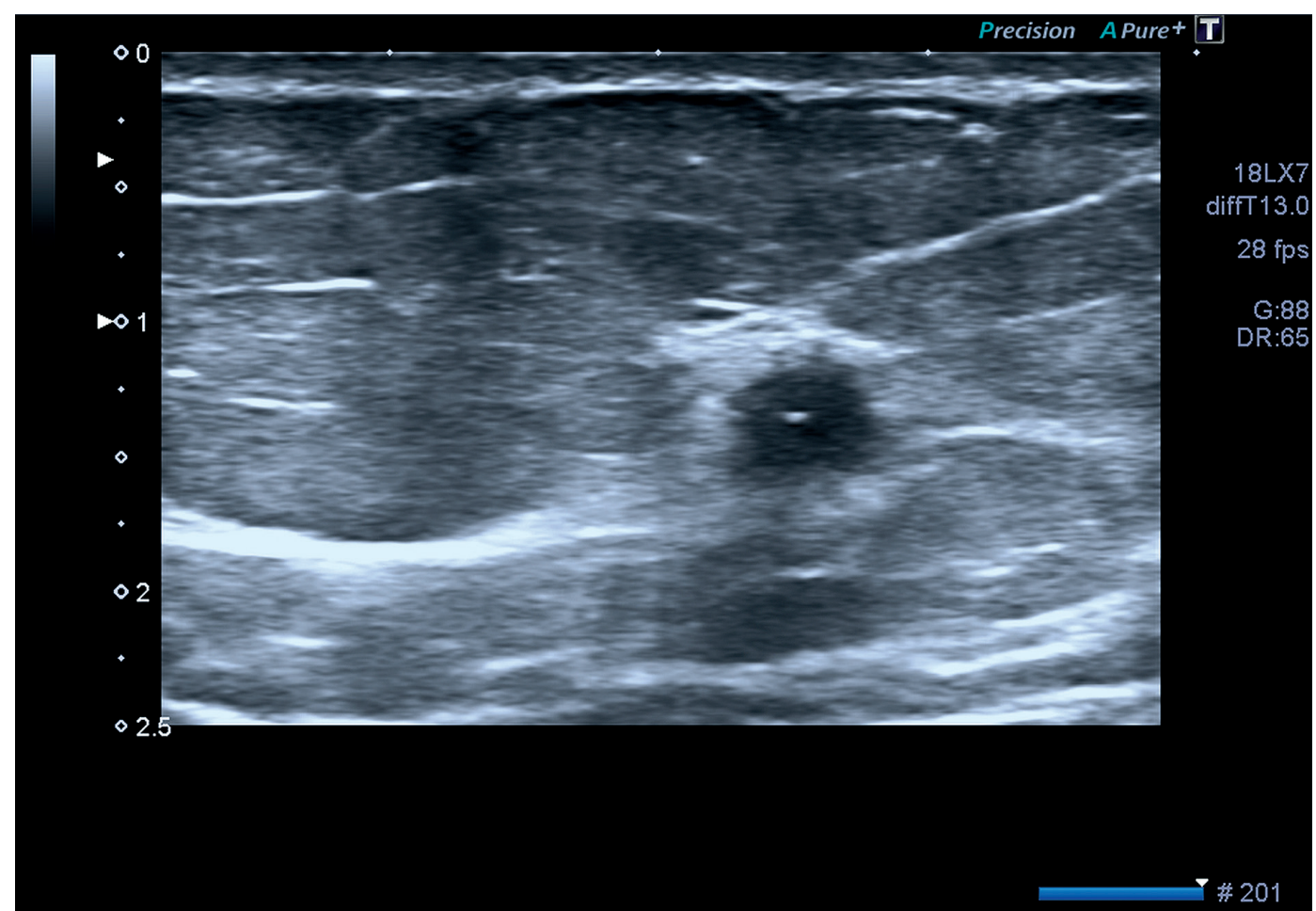

Figure 4. A 5-mm solid breast mass in the upper inner quadrant with malignant appearance. Presence of micro-calcification can be seen. Diagnosis: Invasive ductal carcinoma 
Developing a desmoplastic reaction also causes shadowing in the surrounding tissue rather than the malignant mass itself. Acoustic shadow is commonly seen in invasive ductal carcinoma, which grows slowly and allows a tubular desmoplastic reaction [21].

Hypo-echo dominance showed a PPV of $35.5 \%$, and 256 lesions had this criterion. The positive predictive value and the mass score were very low for this feature. The main reason for this is that fibroadenomas generally appear as hypoechoic and they constitute nearly half of the benign lesions.

The PPV for presence of MC was found to have a ratio of $73.4 \%$. Thirty-four of 46 solid masses with MC were malignant. $\mathrm{MC}$ is a strong indicator of malignancy. The main challenging issue for determining BIRADS score by using US alone is defining MC. The contribution of a MG examination cannot be ignored, especially for patients over age 40 years and with adipose-rich breast tissue. On the other hand, with improvements of US imaging technology in recent years combined with careful examination and optimised imaging presets with sparing long time, even MCs can be detected more than expected (Figure 4). In our study, a careful and long examination was performed for each patient before the biopsy procedure.

The difference between the mean strain ratio of benign and malignant lesions was statistically significant. This result proves that the strain ratio can be used as a malignancy indicator in evaluating solid breast masses. In ROC analysis, the curve obtained was not ideal because of variable strain ratio values (standard deviations). The cutoff value was calculated to be 3.1. Measuring strain ratios is a very user dependent issue, so we believe high standard deviations can be explained in this way. Another potential reason is the strain ratio of adjacent breast tissue. In our experience, measuring the ratio by comparing to adipose or fibroglandular tissue does not affect the results substantially.

There are some articles determining the contribution of elastography to conventional USG findings. Their results can be summarised as follows: Mean SER is higher for malignant masses and the difference is statistically significant. Sonoelastography has a higher specificity than B-mode ultrasound in the differentiation between benign and malignant masses, and it has the potential to reduce biopsies with benign results. Comparing the diagnostic characteristics of the three methods with reference to the definitive histological results for malignant breast lesions, the best diagnostic accuracy is obtained when US, elastography, and colour Doppler imaging are combined [22-24].
In summary, five major grey-scale indicators and SER can be used to determine BIRADS US score. They are: anti-parallel placement to the skin, spiculated and irregular borders, posterior acoustic shadowing (which indicates poor transmission), hypo-echo dominance, and presence of MC.

If BIRADS US scores (EBS) were calculated according to mass score obtained from the PPV of USG malignancy criteria, malignancy ratios were completely concordant with the literature. Therefore, these features can be defined as being competent for evaluation.

A report by Orel et al. [25] evaluated positive predictive values and determined BIRADS scores. They followed the same idea as our research, but the article was concerned with mammographic findings (mass, micro-calcifications, mass with calcifications, asymmetric density, developing density, architectural distortion, architectural distortion with calcifications, and dilated duct).

Another surprising result obtained in the current work was that two of 86 malignant lesions had none of the malignancy indicators mentioned above, and their strain ratios were also below 3.1. Consequently, despite their benign appearance, every solid breast mass still has malignancy potential.

The main limitation of this study was our inability to use shear wave elastography, which is a newer and potentially more objective method. The device we used did not have that option.

\section{Conclusions}

SER can make a contribution to classic US findings, and BIRADS US scores can be objectively determined based on US and elastography features instead of using subjective scoring in evaluating solid breast masses. All solid breast masses still have the possibility to be malignant, even though US and elastography findings indicate the opposite.

\section{Acknowledgements}

We would like to thank our auxiliaries (interventional radiology nurses) Mrs Sibel Açıkbaş and Mrs Ceyda Sivrioğlu.

\section{Conflict of interest}

The authors report no conflict of interest.

\section{References}

1. Khalis M, El Rhazi K, Charaka H, et al. Female Breast Cancer Incidence and Mortality in Morocco: Comparison with Other Countries. Asian Pac J Cancer Prev 2016; 17: 6111-6116.
2. Harvey JA, Mahoney MC, Newell MS, et al. ACR Appropriateness Criteria Palpable Breast Masses. J Am Coll Radiol 2016; 1311S: 31-42. 
3. D'Orsi CJ, Sickles EA, Mendelson EB, et al. (eds.). ACR BI-RADS Atlas, Breast Imaging Reporting and Data System (BI-RADS). $5^{\text {th }} \mathrm{ed}$. American College of Radiology, Reston 2013.

4. Mendelson EB, BohmVelez M, Berg WA, et al. ACR BI-RADS Ultrasound. In: ACR BI-RADS Atlas: Breast Imaging Reporting and Data System. American College of Radiology, Reston 2013.

5. Goddi A, Bonardi M, Alessi S. Breast elastography: a literature review. J Ultrasound 2012; 15: 192-198.

6. Zhao QL, Ruan LT, Zhang H, et al. Diagnosis of solid breast lesions by elastography 5 -point score and strain ratio method. Eur J Radiol 2012; 81: 3245-3249.

7. Fischer T, Peisker U, Fiedor S, et al. Significant differentiation of focal breast lesions: raw data-based calculation of strain ratio. Ultraschall Med 2012; 334: 372-379.

8. Shah VI, Raju U, Chitale D, et al. False-negative core needle biopsies of the breast: an analysis of clinical, radiologic, and pathologic findings in 27 concecutive cases of missed breast cancer. Cancer 2003; 97: 1824-1831.

9. Liberman L, Ernberg LA, Heerdt A, et al. Palpable breast masses: is there a role for percutaneous imaging-guided core biopsy? AJR Am J Roentgenol 2000; 175: 774-787.

10. Ward ST, Shepherd JA, Khalil H. Freehand versus ultrasound-guided core biopsies of the breast: reducing the burden of repeat biopsies in patients presenting to the breast clinic. Breast 2010; 19: 105-108.

11. Pijnappel RM, van Dalen A, Borel Rinkes IH, et al. The diagnostic accuracy of core biopsy in palpable and non-palpable breast lesions. Eur J Radiol 1997; 24: 120-123.

12. Yeow KM, Lo YF, Wang CS, et al. Ultrasound-guided core needle biopsy as an initial diagnostic test for palpable breast masses. J Vasc Interv Radiol 2001; 12: 1313-1317.

13. Philpotts LE, Hooley RJ, Lee CH. Comparison of automated versus vacuum assisted methods for sonography guided core biopsy of breast. Am J Roentgenol 2003; 180: 347-351.
14. Brenner RJ, Fajardo L, Fisher PR, et al. Percutaneous core biopsy of the breast: effect of operator experience and number of samples on diagnostic accuracy. Am J Roentgenol 1996; 166: 341-346.

15. Morrow M. The evaluation of common breast problems. Am Fam Physician 2000; 61: 2371-2378.

16. Klein S. Evaluation of palpable breast masses. Am Fam Physician 2005; 71: 1731-1738.

17. Pruthi S. Detection and evaluation of a palpable breast mass. Mayo Clin Proc 2001; 76: 641-647.

18. Stavros AT, Thickman D, Rapp CL, et al. Solid breast nodules: use of sonography to distinguish between benign and malignant lesions. Radiology 1995; 196: 123-134.

19. Costantini M, Belli P, Lombardi R, et al. Characterization of solid breast masses: use of the sonographic breast imaging reporting and data system lexicon. J Ultrasound Med 2006; 25: 649-659.

20. Hong AS, Rosen EL, Soo MS, et al. BI-RADS for sonography: positive and negative predictive values of sonographic features. Am J Roentgenol 2005; 184: 1260-1265.

21. Shin HJ, Kim HH, Cha JH, et al. Automated Ultrasound of the Breast for Diagnosis: Interobserver Agreement on Lesion Detection and Characterization. Am J Roentgenol 2011; 197: 747-754.

22. Yi A, Cho N, Chang JM, et al. Sonoelastography for 1,786 non-palpable breast masses: diagnostic value in the decision to biopsy. Eur Radiol 2012; 22: 1033-1040.

23. Yoon JH, Ko KH, Jung HK, et al. Qualitative pattern classification of shear wave elastography for breast masses: how it correlates to quantitative measurements. Eur J Radiol 2013; 82: 2199-2204.

24. Botticelli A, Mazzotti E, Di Stefano D, et al. A positive impact of elastography in breast cancer diagnosis: an institutional experience. J Ultrasound 2015; 18: 321-327.

25. Orel SG, Kay N, Reynolds C, et al. BI-RADS categorization as a predictor of malignancy. Radiology 1999; 211: 845-850. 